interface between the oil and water. The team could control the curvature of the interface by changing how oil droplets were attached to the glass, and could generate forces to make the beads group in squares.

Proc. Natl Acad. Sci. http://dx.doi. org/10.1073/pnas.1222196110 (2013)

\section{NEUROSCIENCE}

\section{Romancing the histones}

Pair-bonding in monogamous prairie voles (Microtus ochrogaster, pictured) is linked to chemical modifications of DNA-packaging proteins in the animals' brains.

Zuoxin Wang, Mohamed Kabbaj and their team at Florida State University in Tallahassee studied the brain chemistry of females as they interacted with males. The researchers focused on enzymes that coordinate epigenetic marks on histones, the protein complexes that coil up DNA and regulate gene expression. Females injected with an inhibitor of these enzymes developed a stronger preference for a random male that had been previously placed in their cage than females not injected with the inhibitor. The inhibitor boosted production of receptors for two hormones linked to sexual and maternal behaviours; similar changes are caused by mating.

The authors say these findings are the first to pair the chemistry of coupling with histone regulation.

Nature Neurosci. http://dx.doi. org/10.1038/nn.3420 (2013)

For a longer story on this research, see go.nature.com/fwah2e

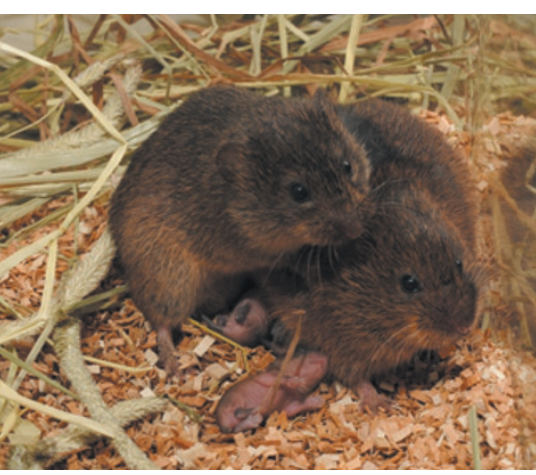

\section{PARTICLE PHYSICS}

\section{A meson unmirrored}

A fourth type of subatomic particle shows imperfect symmetry with its antiparticle.

Charge conjugation parity symmetry holds that an experiment should be indistinguishable from its reflection in a mirror if all particles are replaced with their antiparticles. Since the first exception to this was found in 1964, physicists have been hunting for more particles that violate the principle.

A major collaborative effort co-led by Vincenzo Vagnoni at the Large Hadron Collider at CERN, Europe's particlephysics laboratory near Geneva in Switzerland, identified an asymmetry by tracking decays of the subatomic particles $\mathrm{B}_{\mathrm{s}}{ }_{\mathrm{s}}$ mesons, which are made up of elementary particles called strange quarks and antibottom quarks. The identification of 'CP violation' in these mesons confirmed a prediction of the standard model of particle physics. However, more-drastic violations are required to explain why matter dominates over antimatter in the universe. Phys. Rev. Lett. 110, 221601 (2013)

\section{CLIMATE SCIENCE}

\section{More rain in ozone's absence}

Ozone loss in the stratosphere over Antarctica has increased rainfall in subtropical parts of South America.

Summers in Uruguay, Paraguay, southern Brazil and northern Argentina became markedly wetter over the second half of the twentieth century. To pin down whether this was due to the impact of ozone on atmospheric circulation and precipitation in the region, Paula Gonzales and her group at Columbia University in New York contrasted simulations with and without ozone depletion using six climate models.

COMMUNITY CHOICE

The most viewed papers in science

\title{
Thymus development conserved
}

\section{Q HIGHLY READ \\ on dev.biologists. org in April}

The genetic mechanisms that regulate how an organ of the immune system forms are conserved between mice and humans.

Tucked inside the ribcage and near the heart, the thymus screens out self-attacking white blood cells. Although molecular and gene-expression patterns that govern the formation of the organ can be readily studied in mice, human studies are generally limited to examining tissue samples under a microscope. Thus, similarities between the species are difficult to assess. Clare Blackburn at the University of Edinburgh, UK, and her team used genetic analysis on tissue from human fetuses. They showed that, in contrast to previous findings, the thymus derives from the same embryological structure in humans as it does in mice. In addition, a collection of genes critical to the organ's development are expressed at similar times in both species, further validating the mouse as a model for thymus development Development 140, 2015-2026 (2013)
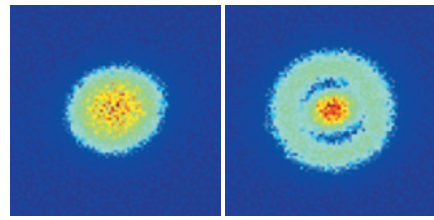

Although results were mixed regarding the impact of greenhouse gases, simulations consistently reproduced observed rainfall trends more accurately when ozone loss was included.

Precipitation in the region should stabilize or decrease as ozone over the Antarctic recovers, the authors suggest. Clim. Dyn. http://dx.doi. org/10.1007/s00382-0131777-x (2013)

\section{Direct view of atomic orbitals}

Electron orbitals of excited hydrogen atoms can be observed directly.

Orbitals lie outside the nucleus and their properties are described by mathematical wavefunctions. These functions are difficult to study because measuring observable components can destroy other

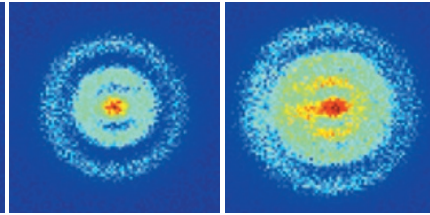

quantum features. Aneta Stodolna at the FOM Institute for Atomic and Molecular Physics in Amsterdam, Marc Vrakking at the Max Born Institute in Berlin and their colleagues designed a quantum microscope to study hydrogen orbitals directly. Their system used tunable lasers to excite electrons in a hydrogen atom placed in an electric field. An electrostatic lens then stretched and magnified the orbitals without disturbing the internal structure - until individual electrons hit a detector. After recording about 50,000 electrons, the team produced images to show the structure of the electron orbital (pictured) of atoms at different excited states.

Phys. Rev. Lett. 110, 213001 (2013)

\section{$\rightarrow$ NATURE.COM}

For the latest research published by Naturevisit:

www.nature.com/latestresearch 